\title{
Mild Versus Conventional Ovarian Stimulation for Poor Responders Undergoing IVF/ICSI
}

\author{
CHARALAMPOS SIRISTATIDIS ${ }^{1}$, GEORGE SALAMALEKIS ${ }^{1}$, KONSTANTINOS DAFOPOULOS $^{2}$, \\ GEORGE BASIOS $^{1}$, PARASKEVI VOGIATZI ${ }^{1}$ and NIKOLAOS PAPANTONIOU ${ }^{1}$ \\ ${ }^{1}$ Assisted Reproduction Unit, Third Department of Obstetrics and Gynecology, Attikon Hospital, \\ Medical School, National and Kapodistrian University of Athens, Athens, Greece; \\ ${ }^{2}$ Assisted Reproduction Unit, Department of Obstetrics and Gynecology, University of Thessaly, \\ School of Health Sciences, Faculty of Medicine, Larissa, Greece
}

\begin{abstract}
Background/Aim: Mild stimulation protocols have been implemented to be offered to subfertile patients who respond poorly to ovarian stimulation. We aimed to compare the efficacy of mild versus conventional gonadotropin-releasing hormone (GnRH)-agonist and antagonist protocols in poor responders undergoing in vitro fertilization/intra-cytoplasmic sperm injection (IVF/ICSI) cycles. Patients and Methods: A total of 58 poorlyresponding patients were divided into two groups: mild

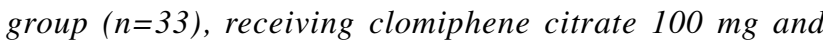
$0.25 \mathrm{mg}$ of cetrorelix with $150 \mathrm{IU}$ of gonadotrophins daily; conventional group $(n=25)$, undergoing the long GnRHagonist or -antagonist protocols. The primary outcome was the number of cumulus oocyte complexes (COCs) retrieved. Results: A lower number of COCs [median (range) $=1$ $(0-4)$ vs. 3 (0-8.4), p<0.001] was retrieved in the mild stimulation compared to the conventional group. Secondary outcomes favored the conventional group, whereas live birth (9.1\% vs. $12 \%)$, clinical pregnancy (12.1\% vs. 20\%) and miscarriage rate (40\% vs. $40 \%)$ were similar in the two groups. Conclusion: Mild ovarian stimulation is inferior to conventional regimes when applied to poor responders undergoing IVF/ICSI, in terms of the numbers of retrieved COCs.
\end{abstract}

Controlled ovarian hyperstimulation $(\mathrm{COH})$ is established as a prerequisite in assisted reproduction technology (ART), as

This article is freely accessible online.

Correspondence to: Charalampos Siristatidis, Rimini 1, Chaidari, Athens 12642, Greece. Mobile: +30 6932294994, e-mail: harrysiri@yahoo.gr

Key Words: Controlled ovarian hyperstimulation, poor responders, recombinant FSH, mild stimulation, clomiphene citrate. it induces coordinated multi-follicular development, leading to a higher yield of oocytes and consequently higher number of embryos to select from, in order to select those with the highest implantation dynamics to increase the chance for a positive outcome in terms of clinical pregnancy.

Subfertile patients with poor ovarian response to exogenous stimulation represent a significant proportion of patients undergoing in vitro fertilization (IVF) and represent a considerable challenge in assisted reproduction due to their low response to $\mathrm{COH}$, the increased cancellation rates observed in these cycles, as well as the decreased probability of pregnancy that accompanies them $(1,2)$. Poor ovarian response has been previously reported to occur in $9-24 \%$ of ART cycles (3). Evidence, to date, indicates that the most efficient approach in managing subfertile poor responders is the individualization of the treatment protocols, based on antral follicle count (AFC) and anti-Mullerian hormone (AMH) values prior to the IVF cycle (4), although the success rate remains low. Difficulties in clinical management arise initially from the complexity surrounding the definition of this group of patients; in this context, in 2011 Ferraretti et al. presented the Bologna criteria in order to characterize this group and adapt its management accordingly (5). Among the various strategies and modified $\mathrm{COH}$ protocols employed over the years towards optimization of management of subfertility, there is no concrete evidence on the advantage of any one stimulation protocol over another $(6,7)$. Some preliminary results demonstrated superiority of the flare-up over the letrozole/antagonist protocols (8), although both gonadotropinreleasing hormone $(\mathrm{GnRH})$-agonist and -antagonist protocols have similar cycle cancellation and clinical pregnancy rates $(9,10)$. A recent comparison among the GnRH-agonist protocols, through a subgroup analysis, including four trials with poor responders, revealed a superiority of long duration GnRH-agonist compared to the short duration (mild) GnRHagonist protocol with regards to clinical pregnancy rates, number of oocytes retrieved, and cancellation rates (11). 
Mild $\mathrm{COH}$ protocols using low doses of gonadotrophins have been implemented in clinical practice, demonstrating significant advantages, including cost effectiveness $(12,13)$, although the expected number of retrieved oocytes is low, usually ranging from two to seven (14). The reduced yield of oocytes is inevitably interlinked with lowered success rates, especially for this group of patients, where the anticipated quality of the gametes is also reduced $(1,2,14-$ 16). Clomiphene citrate is one of the main adjuncts (together with letrozole) used in mild regimes for the ovarian stimulation of poor responders, the definition of whom varies widely.

The rationale of the present study lies within the separate benefits of mild stimulation through lower gonadotrophin doses, the lowered cost that accompanies the treatment, together with its suspected equality in terms of effectiveness to the conventionally used protocols for ovarian stimulation. In this framework, the purpose of this case-control study was to compare a clomiphene citrate-based mild stimulation regime with the conventional GnRH-agonist and -antagonist protocols in poor responders defined by the Bologna criteria.

\section{Patients and Methods}

Patient population and study design. This case-control study was conducted at the Assisted Reproductive Unit of the Third Department of Obstetrics and Gynecology, Attikon Hospital, Medical School, National and Kapodistrian University of Athens, Greece, during the period from March 2011 to September 2015. The initial design was a single center, prospective, randomized study (RCT), to be conducted to the referral University Hospital, registered at clinicalstudies.gov on March 8th, 2011 (NCT01319708). The trial protocol was approved by the Scientific Board (protocol no.519/23-02-2011) and Bioethics Committee of the Hospital (approval no. 3/04-03-2011). Due to problems in the randomization process, the relevant data are preferably analyzed as a case-control study in order to contribute the outcomes of the study, although in an altered nature.

Inclusion criteria followed the Bologna criteria (5). Only fresh ejaculated sperm was used, while no preimplantation genetic screening was allowed. Following clinical evaluation along with previous medical and reproductive history, participants were categorized according to subfertility factor as follows: male, female (tubal infertility, ovulatory dysfunction and endometriosis) and unexplained infertility. Further demographic parameters were recorded along with the respective hormonal profiles: age, body mass index (BMI), smoking status, parity, basal AMH and folliclestimulating hormone (FSH) levels, AFC, type (primary/secondary) and duration of subfertility.

The exclusion criteria for participation in the study were: increased basal level of FSH at day 3 of the menstrual cycle ( $>20 \mathrm{IU} / \mathrm{l})$, increased BMI $>35 \mathrm{~kg} / \mathrm{m}^{2}$, history of endocrine or metabolic disorders, ovarian cystectomy or oophorectomy, severe endometriosis or severe azoospermia.

Patients were allowed to participate in the study once and written informed consent was obtained from all participants following consultation and before the initiation of the treatment cycles.
Fifty-eight participants that met the inclusion criteria were enrolled in the study. Of them, 33 were treated with a mild regimen, consisting the study group. The control group consisted of 25 subfertile participants with matching baseline and demographic characterisitcs, conforming with the same inclusion criteria and was treated with a long-duration GnRH-agonist or GnRH-antagonist protocol. Our analysis was performed to provide a direct comparison of the study and control groups. In order to avoid potential sources of bias, we included participants who were referred to the Unit consecutively.

Ovarian stimulation protocol. For the long-duration GnRH-agonist protocol, triptorelin [0.1 mg Gonapeptyl (Ferring Pharmaceutical Hellas A.E., Athens, Greece) or Arvekap (Ipsen Pharma, France)] administration started subcutaneously daily at the midluteal phase of the menstrual cycle. For the GnRH-antagonist protocol, ovarian stimulation began on the second day of the cycle and the antagonist, either cetrorelix (Cetrotide; Merck Serono Europe Limited, London, UK) or ganirelix (Orgalutran; NV Organon, Oss, the Netherlands) was initiated as soon as the leading follicle reached a diameter of $\geq 14 \mathrm{~mm}$ on average, and continued up to the day of human chorionic gonadotrophin (hCG) administration. For the mild stimulation protocol, $100 \mathrm{mg}$ of clomiphene citrate was administered starting on day 2 up to day 6 of the cycle; 150 IU of gonadotrophin (r-FSH) together with a fixed dose of cetrorelix or ganirelix were initiated as soon as the leading follicle reached a diameter of $\geq 14 \mathrm{~mm}$ on average and continued up to the day of hCG administration.

Once pituitary down-regulation and ovarian suppression were achieved, ovarian stimulation with exogenous gonadotrophins was initiated, while GnRH agonist dose was reduced to half and continued concomitantly until the day of hCG administration. Recombinant FSH in the form of either follitropin alpha (Gonal-F; Merck Serono Europe Ltd) or follitropin beta (Puregon; NV Organon) was administered subcutaneously. Starting dose was $300 \mathrm{IU}$ (with the maximum set at $450 \mathrm{IU}$ ) and was adjusted according to individual participant's response to stimulation, after day 6 of cycle monitoring. Transvaginal ultrasonography was repeated every 2-4 days. When two or more follicles reached a mean diameter of $17 \mathrm{~mm}$, human chorionic gonadotrophin $[10,000$ IU Pregnyl (NV Organon)] was administered intramuscularly prior to transvaginal ultrasound-guided oocyte retrieval (OR) $36-38 \mathrm{~h}$ later. In case of monofollicular development, patients were allowed to proceed to OR after their written informed consent. Intracytoplasmic sperm injection (ICSI) was performed in all the reported cases. Depending on the embryo quality, embryo transfer was performed either 2 or 3 days following OR, whereas the maximum number of embryos transferred was three, and in each case separately, in accordance with Hellenic legislation.

Luteal-phase support in all participants was achieved by transvaginal administration of micronized progesterone in the form of vaginal suppositories (Utrogestan, vaginal tablets, $200 \mathrm{mg}$ tid; Basins Iscovesco., Paris, France), immediately starting from the afternoon of the day of OR.

Outcome measures. The primary outcome measure was the number of cumulus oocyte complexes (COCs) retrieved at the day of the OR. Secondary outcome measures included duration of stimulation, total dose of gonadotropins administered, number of follicles measuring 14-15, 16-17 and $\geq 18 \mathrm{~mm}$ along with endometrial 
thickness on the day of triggering, number of metaphase II (MII) oocytes, fertilization rate and fertilized oocytes, transferred and frozen embryos, cycle cancellation and failed fertilization rates, positive $\beta$-hCG, clinical pregnancy, miscarriage and live birth per randomized patient.

Clinical pregnancy was defined as the confirmation of the presence of the intrauterine sac with fetal heart activity through transvaginal ultrasound scan at seven weeks of gestation, following a positive $\beta$-hCG test. Cycle cancellation was forced in cases where premature ovulation was evident by the loss of follicle(s) prior to $\mathrm{OR}$, and in cases where there were no COCs retrieved or no embryos available for transfer. The miscarriage rate was defined as pregnancy loss earlier than 20 weeks of gestation, following a positive serum assay for pregnancy. Embryo quality was assessed according to morphological criteria based on the overall blastomere number and appearance and the degree of fragmentation (17).

Power calculation - sample size. The sample size was estimated based on the number of COCs retrieved. In this context, and based on previous study designs $(16,20)$, we agreed on a difference of two COCs retrieved between the two groups with a standard deviation of 2.5 . By using two-sided t-test, with $80 \%$ power, 25 patients in each group would be required, assuming a significance level of 0.05 . Considering possible dropouts, we aimed to enroll around 30 patients in each arm. The statistical software package used for sample-size calculation was PASS v.11 (NCSS, LLC, Kaysville, UT, USA).

Statistical analysis. Analysis per intention-to-treat (ITT) was performed by including participants according to their initial treatment intent. Thus, all patients who started treatment were included in the analysis regardless of whether they proceeded to OR or not. In cases where OR was cancelled, participants were considered as having zero retrieved COCs.

Categorical variables were expressed as percentages of the corresponding population and compared using the chi-square test. Continuous variables were assessed for normal distribution by the Kolmogorov-Smirnov test. Data were normally distributed for both groups and comparisons were performed with parametric test ( $t$-test) for the following variables: age, BMI, FSH, AMH, AFC, duration of stimulation, dose of gonadotrophins, endometrial thickness, number of oocytes retrieved, number of MII oocytes, number of fertilized oocytes and fertilization rate. For the remaining comparisons, where each variable was not normally distributed for at least one group, non-parametric test (Mann-Whitney $U$-test) was used. All continuous variables were expressed as medians (with 95\% confidence intervals). Differences were considered as statistically significant if the null hypothesis was rejected with $>95 \%$ confidence $(p<0.05)$. Statistical analysis was performed using the SPSS v.20 statistical software package (IBM Corp., Armonk, NY, USA).

\section{Results}

The study cohort consisted of 58 patients, characterized as poor responders according to the Bologna criteria (5). The study lasted for more than 4 years (June 2011 until November 2015), and during this period, no significant changes in terms of personnel /equipment or pregnancy rates occurred. Patient characteristics were comparable between the two groups and are presented in Table I. Outcomes and cycle characteristics are summarized in Table II. Stimulation duration did not differ between groups, as the time of clomiphene citrate use was co-calculated. In contrast, the total gonadotrophin dose was statistically different between groups $[1,050(95 \% \mathrm{CI}=0$ $2,490) v s .4,040$ (95\% CI=1,800-6,795) IU, $p<0.001]$.

A significantly higher number of follicles in the category of $14-15 \mathrm{~mm}$ and $18 \mathrm{~mm}$, although not for $16-17 \mathrm{~mm}$, was observed in the control group. There was no monofollicular development in either of the two groups. Similarly a higher number of COCs and MII oocytes [1 (95\% CI=0-4) vs. 3 (95\% CI=0-8.4), $p<0.001$ and $1(95 \% \mathrm{CI}=0-4)$ vs. $2(95 \%$ $\mathrm{CI}=0-7.4), p=0.001$, respectively], and number of fertilized oocytes, cleaved, transferred and frozen embryos (all pvalues <0.05) was observed in the control group. The endometrial thickness on the day of hCG administration was significantly lower in the study group compared to the control group $[7.8(95 \% \mathrm{CI}=4.1-12)$ vs. 10 (95\% CI=5.113.6) $\mathrm{mm}, p=0.015]$ and cancellation rates were significantly higher [36.4\% (95\% CI=19-53.7) vs. $12 \%$ (95\% CI=1.725.7), $p=0.036]$. All cancellations occurred due to the failed retrieval of COCs during OR for both groups, although there was at least a leading follicle of over $16 \mathrm{~mm}$ at triggering in all cases. There were no cases of failed fertilization.

There was no difference between groups with regards to $\beta$-hCG-positivity [15.2\% (95\% CI=2.2-28.1) vs. $20 \%(95 \%$ $\mathrm{CI}=3.1-36.9), p=0.628]$, clinical pregnancy $[12.1 \%(95 \%$ $\mathrm{CI}=4-23.9) v s .20 \%(95 \% \mathrm{CI}=3.1-36.9), p=0.412]$, and live birth $[9.1 \%(95 \% \mathrm{CI}=1.3-19.4)$ vs. $12 \%(95 \% \mathrm{CI}=1.7-25.7)$, $p=0.719]$ rates, nor to miscarriages [40\% (95\% CI=28-100) vs. $40 \%(95 \% \mathrm{CI}=28-100), p=1.000]$, although the study was under-powered to detect such differences.

\section{Discussion}

In this case-control study, we evaluated the efficiency of the mild stimulation protocol for IVF over the conventional GnRH long agonist/antagonist protocols in a group of subfertile women with poor ovarian response, according to the Bologna criteria. As the primary outcome, we used more than two COCs to determine the sample size. The outcomes examined included parameters of the stimulation processes in preparation for IVF, as well as the embryological and clinical outcome following ART. The number of COCs retrieved favored the conventional protocols. In addition, gonadotrophin intake was significantly lower for the women that received the mild protocol; in contrast, our results demonstrated that clinical pregnancy, miscarriage and live birth rates were similar for both protocols, although the study was underpowered to detect such differences.

Clomiphene citrate (18-21) and letrozole (22-26) are the two most common pharmaceutical compounds administered 
Table I. Baseline characteristics of the mild (group 1) and conventional regimen (group 2) groups. Numeric data are presented as medians (with 95\% confidence interval). Percentages are presented as absolute numbers (with 95\% confidence interval). A value of p<0.05 was considered statistically significant.

\begin{tabular}{lccc}
\hline Characteristic & Group 1 (n=33) & Group 2 (n=25) & $p$-Value \\
\hline Age (years) & $39.0(34.0-45.0)$ & $40.0(33.0-45.0)$ & 0.244 \\
BMI (kg/m $\left.{ }^{2}\right)$ & $23.5(20.0-29.1)$ & $24.8(20.0-33.4)$ & 0.173 \\
FSH (IU/I) & $8.9(3.5-17.8)$ & $8.2(3.7-19.4)$ & 0.745 \\
AMH (ng/ml) & $1.6(0.3-7.8)$ & $1.7(0.2-4.7)$ & 0.205 \\
AFC & $3.0(2.0-7.0)$ & $4.0(1.0-9.7)$ & 0.062 \\
Smoking (Yes) & $33.3 \%(16.4-50.3)$ & $48.0 \%(27.0-69.0)$ & 0.258 \\
Parity (Yes) & $24.2 \%(8.8-39.7)$ & $28.0 \%(9.1-46.9)$ & 0.746 \\
Primary subfertility & $66.7 \%(49.7-83.6)$ & $52.0 \%(31.0-73.0)$ & 0.258 \\
Male factor & $39.4 \%(21.8-57.0)$ & $40.0 \%(19.4-60.6)$ & 0.963 \\
Tubal factor & $30.3 \%(13.8-46.9)$ & $28.0 \%(9.1-46.9)$ & 0.849 \\
Ovulatory factor & $6.1 \%(0.25-14.7)$ & $12.0 \%(1.7-25.7)$ & 0.425 \\
Endometriosis & $12.1 \%(4.0-23.9)$ & $12.0 \%(1.7-25.7)$ & 0.989 \\
Unexplained infertility & $15.2 \%(2.2-28.1)$ & $12.0 \%(1.7-25.7)$ & 0.730 \\
Duration of subfertility (years) & $3.0(1.0-8.6)$ & $2.0(1.0-8.7)$ & 0.312 \\
\hline
\end{tabular}

AFC: Antral follicle count; AMH: anti-Mullerian hormone; BMI: body mass index; FSH: follicle-stimulating hormone.

Table II. Characteristics of the stimulation cycle and clinical outcomes of the mild (group 1) and conventional regimen (group 2) groups. Numeric data are presented as medians (with 95\% confidence interval). Percentages are presented as absolute numbers (with $95 \%$ confidence interval). A value of $p<0.05$ was considered statistically significant.

\begin{tabular}{lccc}
\hline Characteristic & Group 1 (n=33) & Group 2 (n=25) & $p$-Value \\
\hline Dose of gonadotropins (IU) & $1050(0-2490)$ & $4040(1800-6795)$ & $<0.001$ \\
Duration of stimulation (days) & $11.0(5.0-15.0)$ & $12.0(9.0-16.0)$ & 0.140 \\
No. of follicles (14-15 mm) & $0.0(0.0-1.3)$ & $1.0(0.0-3.7)$ & 0.003 \\
No. of follicles (16-17 mm) & $1.0(0.0-3.0)$ & $1.0(0.0-4.7)$ & 0.832 \\
No. of follicles (18 mm) & $1.0(0-4.0)$ & $2.0(0.3-4.7)$ & 0.007 \\
No. of COCs & $1.0(0.0-4.0)$ & $3.0(0.0-8.4)$ & $<0.001$ \\
No. of MII oocytes & $1.0(0.0-4.0)$ & $2.0(0.0-7.4)$ & 0.001 \\
Fertilization rate & $1.0(0.6-1.0)$ & $1.0(0.5-1.0)$ & 0.342 \\
Cancellation rate & $36.4 \%(19.0-53.7)$ & $12 \%(1.7-25.7)$ & 0.036 \\
Endometrial thickness (mm) & $7.8(4.1-12.0)$ & $10.0(5.1-13.6)$ & 0.015 \\
No. of embryos & $1.0(0.0-3.3)$ & $2.0(0.0-6.4)$ & 0.008 \\
No. of transferred embryos & $1.0(0.0-2.3)$ & $2.0(0.0-3.0)$ & 0.003 \\
No. of frozen embryos & $0.0(0.0-1.3)$ & $0.0(0.0-3.0)$ & 0.046 \\
Positive $\beta$-HCG & $15.2 \%(2.2-28.1)$ & $20.0 \%(3.1-36.9)$ & 0.628 \\
Miscarriage & $40.0 \%(28.0-100.0)$ & $40.0 \%(28.0-100.0)$ & 1.000 \\
Clinical pregnancy & $12.1 \%(4.0-23.9)$ & $20.0 \%(3.1-36.9)$ & 0.412 \\
Live birth & $9.1 \%(1.3-19.4)$ & $12.0 \%(1.7-25.7)$ & 0.719 \\
\hline
\end{tabular}

COCs: Cumulus oocyte complexes; MII: metaphase II; $\beta$-HCG: b-human chorionic gonadotropin.

for mild ovarian stimulation in poor responders. Nine trials have been published to date utilizing either drug in a mild regime but with varying inclusion criteria in the context of poor ovarian response, thus a differing definition of poor responders complicates an attempted direct comparison of the studies (18-26). For the purpose of this study, we provide a comparison of our findings with the published data on previous studies using clomiphene citrate (18-21) as the different action cascade of the chemical compounds in letrozole, for example, could have potentially affected the investigated outcomes.

The dose of gonadotrophins used for $\mathrm{COH}$ in the control group was three-fold higher than that of the study group and this finding is in agreement with all four trials published to 
date, that utilized clomiphene citrate for their mild regime (18-21). The increased gonadotrophin dose had a direct impact on the number of retrieved COCs and the number of mature oocytes, a finding consistent with one of the studies previously published (20), while others report similar (18, 21) and one lower rates (19). In addition, mild stimulation with clomiphene citrate was linked with an increased cancellation rate due to failed retrieval of oocytes, as also denoted in two studies $(18,20)$, whereas in the remaining two, cancellation rates did not significantly differ between groups $(19,21)$. Fertilization rates were high and similar under the two regimes used, probably demonstrating a good efficiency of the Embryology Laboratory practice and performance of ICSI in all cases. This finding is also in agreement with previous work (18-21), probably denoting the irrelevance of the protocol to the embryological procedures and potentially with to the quality of the oocytes obtained. Endometrial thickness was higher in the control group, an observation probably owing to the anti-estrogenic effect of clomiphene citrate on the endometrium of these patients, a finding that was similar to those of the largest RCTs $(19,20)$. As expected, higher doses of gonadotrophins in the control group resulted in greater number of embryos available for transfer and the number of transferred embryos. These findings agree with those of the largest RCT conducted (20), while the rest report similar (19) or even lower (21) numbers of embryos.

In contrast, positive $\beta$-hCG, clinical pregnancy and live birth rates were low, but similar for both groups studied, as was the miscarriage rate, which appeared to be increased in both regimes. These results might possibly be attributed to the otherwise anticipated low pregnancy rates in this category of patients with compromised ovarian response (16, $27,28)$, added to the observation that a higher dose of gonadotropin does lead to improved implantation rates (29). Regarding clinical pregnancy rates, our findings are in agreement with the existing trials (19-21); notably, a difference of $8 \%$, favoring the conventional protocol did not manage to reach statistical significance, as one would expect the higher number of COCs to result in such a difference. Regarding miscarriage, the currently presented results were in accordance with the results of the largest RCT (20). Live birth rates were reported by only one trial (18), and did not differ between the two protocols, similarly to our study.

The importance of the reported results lies in the fact that poor responders have also an anticipated poor outcome in conventional IVF protocols and in many cases there are no embryos available for transfer. There are a number of previously reported benefits for mild ovarian stimulation in normal responders, including the short duration of the protocol, clinical simplicity of use, reduced patient discomfort, and lowered costs and accompanied risk $(12,30$, 31 , reduced incidence of mosaicism and chromosomal abnormalities resulting in improved embryo dynamics (32), and a qualitatively lower interference in the natural selection of good-quality oocytes (33). The increased risk of cycle cancellation and the inevitable initiation of new treatment cycles are pictured as the main disadvantages over the standardization of the mild protocol in clinical practice. In this regard, it should be presumed that the risk of cancellation, although lower, still exists for patients under the conventional protocols, in addition to the fact that cycle cancellation prior to OR would otherwise protect the patient from unnecessary procedures that pose medical risks themselves.

The current study was designed and initiated 4 months prior to the actual publication of the Bologna criteria (5). The inclusion criteria used coincided with the published criteria and no modifications or adjustments were performed and were mainly based on participants' advanced age, previous poor ovarian response to stimulation and AFC or $\mathrm{AMH}$ levels. There has been much criticism over these criteria, mainly due to the various mechanisms and risk factors linked with poor ovarian reserve, importantly expressed by the same authors $(34,35)$; of note, poor responders are not a homogenous group of the subfertile population (36).

A certain limitation of the current study is the lowered clinical value of the presented results attributed to the lack of randomization and the small cohort size. By assuming the number of COCs anticipated in OR in comparison to the conventional approach, our primary outcome shifted from live birth, a parameter that adds to the significance of the results of the study when considering clinical practice. By reporting live birth as a primary outcome, a large number of participants would be required $(\geq 200)$ and this could solely be achieved in a multicenter setting, as stated in a recent trial (16). Another limitation might be the existence of two conventional protocols, representing the control group. This could be justified by the standard policy of our Unit to use both protocols for this category of patients and due to the fact that both protocols appear to be equal in terms of success rates when applied to poor responders $(5,8,9,37)$. We also fully acknowledge the difference in terms of oocyte quality and quantity when stimulated between poor responders at a young and advanced age, or those with different phenotypes: again here, the sample size warranted would be much higher and the conducting of this study much longer. Finally, the results described are linked to the specific protocol used, as there are trials where gonadotrophins starting at the early follicular phase, and GnRH antagonists at a later date or not at all, reporting better outcomes $(20,29$, $38,40,41)$.

In conclusion, in poor responders to ovarian stimulation as defined by the Bologna criteria, the number of COCs retrieved following ovarian stimulation with the mild protocol, as studied using clomiphene citrate, was lower 
compared to the use of high doses of gonadotrophins through long-duration and GnRH-antagonist protocols. Moreover, the resulting cancellation rates and embryos produced favor the conventional regimes. A multicenter collaboration in a randomized trial would ideally provide clinical significance to the currently reported information, in the context of recruiting a greater population size and establishing live birth rate as the primary outcome.

\section{Conflicst of Interest}

All Authors declare no conflict of interest.

\section{Acknowledgements}

The Authors would like to thank the administrative and clinical staff of the Assisted Reproduction Unit of Attikon University Hospital for their valuable contribution, and especially Tereza Vrantza, the embryologist of the Unit, for her contribution to the collection of data, apart from her unique laboratory work in all the cases studied.

\section{References}

1 Kolibianakis EM, Venetis CA, Diedrich K, Tarlatzis BC and Griesinger G: Addition of growth hormone to gonadotrophins in ovarian stimulation of poor responders treated by in vitro fertilization: a systematic review and meta-analysis. Hum Reprod Update 15: 613-622, 2009.

2 Kyrou D, Kolibianakis EM, Venetis CA, Papanikolaou EG, Bontis $\mathbf{J}$ and Tarlatzis BC: How to improve the probability of pregnancy in poor responders undergoing in vitro fertilization: a systematic review and meta-analysis. Fertil Steril 91: 749-766, 2009.

3 Fasouliotis S, Simon A and Laufer N: Evaluation and treatment of low responders in Assisted Reproductive Technology: A challenge to meet. J Assist Reprod Genet 17: 357-373, 2000.

4 La Marca A and Sunkara SK: Individualization of controlled ovarian stimulation in IVF using ovarian reserve markers: from theory to practice. Hum Reprod Update 20: 124-140, 2014.

5 Ferraretti AP, La Marca A, Fauser BC, Tarlatzis B, Nargund G and Gianaroli L; ESHRE working group on Poor Ovarian Response Definition: ESHRE consensus on the definition of 'poor response' to ovarian stimulation for in vitro fertilization: the Bologna criteria. Hum Reprod 26: 1616-1624, 2011.

6 Pandian Z, McTavish AR, Aucott L, Hamilton MP and Bhattacharya S: Interventions for 'poor responders' to controlled ovarian hyper stimulation $(\mathrm{COH})$ in in vitro fertilisation (IVF). Cochrane Database Syst Rev 1: CD004379, 2010.

7 Polyzos NP and Devroey P: A systematic review of randomized trials for the treatment of poor ovarian responders: Is there any light at the end of the tunnel? Fertil Steril 96: 1058-1061, 2011.

8 Song Y, Li Z, Wu X, Wang X, Xiao J and Wang B: Effectiveness of the antagonist/letrozole protocol for treating poor responders undergoing in vitro fertilization/intracytoplasmic sperm injection: a systematic review and meta-analysis. Gynecol Endocrinol 30: 330-334, 2014.

9 Xiao J, Chang S and Chen S: The effectiveness of gonadotropinreleasing hormone antagonist in poor ovarian responders undergoing in vitro fertilization: a systematic review and metaanalysis. Fertil Steril 100: 1594-1601, 2013.

$10 \mathrm{Pu} \mathrm{D}, \mathrm{Wu} \mathrm{J}$ and Liu J: Comparisons of GnRH antagonist versus GnRH agonist protocol in poor ovarian responders undergoing IVF. Hum Reprod 26: 2742-2749, 2011.

11 Siristatidis CS, Gibreel A, Basios G, Maheshwari A and Bhattacharya S: Gonadotrophin-releasing hormone agonist protocols for pituitary suppression in assisted reproduction. Cochrane Database Syst Rev 1: CD006919, 2015.

12 Siristatidis C, Trivella M, Chrelias C, Sioulas VD, Vrachnis N and Kassanos D: A short narrative review of the feasibility of adopting mild ovarian stimulation for IVF as the current standard of care. Arch Gynecol Obstet 286: 505-510, 2012.

13 Nargund G, Fauser BC, Macklon NS, Ombelet W, Nygren K and Frydman R; Rotterdam ISMAAR Consensus Group on Terminology for Ovarian Stimulation for IVF: The ISMAAR proposal on terminology for ovarian stimulation for IVF. Hum Reprod 22: 2801-2804, 2007.

14 Lefebvre J, Antaki R, Kadoch IJ, Dean NL, Sylvestre C, Bissonnette F, Benoit J4, Ménard S and Lapensée L: 450 IU vs. 600 IU of gonadotropins for controlled ovarian stimulation in poor responders: a randomized controlled trial. Fertil Steril 104: 1419-1425, 2015.

15 Siristatidis C, Vogiatzi P, Bettocchi S, Basios G, Mastorakos G and Vrachnis N: Transvaginal ovarian trauma, poor responders and improvement of success rates in IVF; anecdotal data and a hypothesis. Med Hypotheses 83: 227-231, 2014.

16 Kolibianakis EM, Venetis CA, Bosdou JK, Zepiridis L, Chatzimeletiou K, Makedos A, Masouridou S, Triantafillidis S, Mitsoli A and Tarlatzis BC: Corifollitropin alpha compared with follitropin beta in poor responders undergoing ICSI: a randomized controlled trial. Hum Reprod 30: 432-440, 2015.

17 Ziebe S, Petersen K, Lindenberg S, Andersen AG, Gabrielsen A and Andersen AN: Embryo morphology or cleavage stage: How to select the best embryos for transfer after in vitro fertilization. Hum Reprod 12: 1545-1549, 1997.

18 Fujimoto A, Harada M, Hirata T, Osuga Y and Fujii T: Efficacy of clomiphene citrate supplementation to conventional GnRH antagonist protocols in poor responders undergoing assisted reproductive technology - a prospective randomized trial. Fertil Steril 102(3): e65, 2014.

19 Karimzadeh MA, Mashayekhy M, Mohammadian F and Moghaddam FM: Comparison of mild and microdose $\mathrm{GnRH}$ agonist flare protocols on IVF outcome in poor responders. Arch Gynecol Obstet 283: 1159-1164, 2011.

20 Revelli A, Chiadò A, Dalmasso P, Stabile V, Evangelista F, Basso G and Benedetto C: "Mild" vs. "long" protocol for controlled ovarian hyperstimulation in patients with expected poor ovarian responsiveness undergoing in vitro fertilization (IVF): a large prospective randomized trial. J Assist Reprod Genet 31: 809-815, 2014.

21 Youssef MAFM, Khalil I, Khattab S, Aboulfotouh I, van Wely $M$ and van der Veen F: Mild ovarian stimulation for women with poor ovarian response undergoing IVF/ICSI treatment cycles; randomized controlled study. Fertil Steril 96(3): S263, 2011.

22 Davar R, Oskouian H, Ahmadi S and Firouzabadi RD: GnRH antagonist/letrozole versus microdose GnRH agonist flare protocol in poor responders undergoing in vitro fertilization. Taiwan J Obstet Gynecol 49: 297-301, 2010. 
23 Lee VC, Chan CC, Ng EH, Yeung WS and Ho PC: Sequential use of letrozole and gonadotrophin in women with poor ovarian reserve: a randomized controlled trial. Reprod Biomed Online 23: 380-388, 2011

24 Mohsen IA and El Din RE: Minimal stimulation protocol using letrozole versus microdose flare-up GnRH agonist protocol in women with poor ovarian response undergoing ICSI. Gynecol Endocrinol 29: 105-108, 2013.

25 Ozmen B, Sönmezer M, Atabekoglu CS and Olmus H: Use of aromatase inhibitors in poor-responder patients receiving GnRH antagonist protocols. Reprod Biomed Online 19: 478-485, 2009.

26 Schoolcraft WB, Surrey ES, Minjarez DA, Stevens JM and Gardner DK: Management of poor responders: Can outcomes be improved with a novel gonadotropin-releasing hormone antagonist/letrozole protocol? Fertil Steril 89: 151-156, 2008.

27 Ulug U, Ben-Shlomo I, Turan E, Erden HF, Akman MA and Bahceci M: Conception rates following assisted reproduction in poor responder patients: a retrospective study in 300 consecutive cycles. Reprod Biomed Online 6: 439-443, 2003.

28 Kahraman K, Berker B, Atabekoglu CS, Sonmezer M, Cetinkaya E, Aytac R and Satiroglou H: Microdose gonadotropin-releasing hormone agonist flare-up protocol versus multiple dose gonadotropin-releasing hormone antagonist protocol in poor responders undergoing intracytoplasmic sperm injection-embryo transfer cycle. Fertil Steril 91: 2437-2444, 2009.

29 Devroey P, Bourgain C, Macklon NS and Fauser BC: Reproductive biology and IVF: ovarian stimulation and endometrial receptivity. Trends Endocrinol Metab 15: 84-90, 2004.

30 Fauser BC, Nargund G, Andersen AN, Norman R, Tarlatzis B, Boivin $\mathrm{J}$ and Ledger W: Mild ovarian stimulation for IVF: 10 years later. Hum Reprod 25: 2678-2684, 2010.

31 Polinder S, Heijnen EM, Macklon NS, Habbema JD, Fauser BJ and Eijkemans MJ: Cost-effectiveness of a mild compared with a standard strategy for IVF: a randomized comparison using cumulative term live birth as the primary endpoint. Hum Reprod 23: 316-323, 2008.

32 Baart EB, Martini E, Eijkemans MJ, Van Opstal D, Beckers NG, Verhoeff, Macklon NS and Fauser BC: Milder ovarian stimulation for in vitro fertilization reduces aneuploidy in the human preimplantation embryo: a randomized controlled trial Hum Reprod 22: 980-988, 2007.

33 Verberg MF, Eijkemans MJ, Macklon NS, Heijnen EM, Baart EB, Hohmann FP, Fauser BC and Broekmans FJ: The clinical significance of the retrieval of a low number of oocytes following mild ovarian stimulation for IVF: a meta-analysis. Hum Reprod Update 15: 5-12, 2009.
34 Ferraretti AP and Gianaroli L: The Bologna criteria for the definition of poor ovarian responders: Is there a need for revision? Hum Reprod 29: 1842-1845, 2014.

35 Venetis CA: The Bologna criteria for poor ovarian response: the good, the bad and the way forward. Hum Reprod 29: 1839-1841, 2014.

36 Oudendijk JF, Yarde F, Eijkemans MJ, Broekmans FJ and Broer SL: The poor responder in IVF: Is the prognosis always poor?: A systematic review. Hum Reprod Update 18: 1-11, 2012.

37 Sunkara SK, Coomarasamy A, Faris R, Braude P and Khalaf Y: Long gonadotropin-releasing hormone agonist versus short agonist versus antagonist regimens in poor responders undergoing in vitro fertilization: a randomized controlled trial. Fertil Steril 101: 147-153, 2014.

38 Figueiredo JB, Nastri CO, Vieira AD and Martins WP: Clomiphene combined with gonadotropins and $\mathrm{GnRH}$ antagonist versus conventional controlled ovarian hyperstimulation without clomiphene in women undergoing assisted reproductive techniques: systematic review and meta-analysis. Arch Gynecol Obstet 287: 779-790, 2013.

39 Kato K, Takehara Y, Segawa T, Kawachiya S, Okuno T, Kobayashi T, Bodri D and Kato O: Minimal ovarian stimulation combined with elective single embryo transfer policy: agespecific results of a large, single-centre, Japanese cohort. Reprod Biol Endocrinol 10: 35, 2012.

$40 \mathrm{Li} \mathrm{Y,} \mathrm{Yang} \mathrm{W,} \mathrm{Chen} \mathrm{X,} \mathrm{Li} \mathrm{L,} \mathrm{Zhang} \mathrm{Q} \mathrm{and} \mathrm{Yang} \mathrm{D:} \mathrm{Comparison}$ between follicular stimulation and luteal stimulation protocols with clomiphene and HMG in women with poor ovarian response. Gynecol Endocrinol 32: 74-77, 2016.

41 Zhang JJ, Merhi Z, Yang M, Bodri D, Chavez-Badiola A, Repping S and van Wely M: Minimal stimulation IVF $v s$. conventional IVF: a randomized controlled trial. Am J Obstet Gynecol 214: 96.e1-8, 2016.
Received November 30, 2016

Revised January 21, 2017

Accepted January 24, 2017 